\title{
The interface between metallurgy and mechanics in material performance
}

\author{
M. N. James \\ School of Marine Science \& Engineering, University of Plymouth, Plymouth (UK) \\ Department of Mechanical Engineering, Nelson Mandela Metropolitan University, Port Elizabeth (South Africa) \\ mjames@plymouth.ac.uk. \\ M. Newby \\ School of Marine Science \& Engineering, University of Plymouth, Plymouth (UK) \\ Eskom Research and Innovation Centre, Rosherville, Johannesburg, South Africa
}

\begin{abstract}
This paper considers an important topic, and one that is often poorly understood or misinterpreted, but which is a determining factor in many aspects of the service performance of metals (and other materials). Engineering components and structures must, of necessity, provide a bridge between the macroscopic, homogeneous and generally continuum aspects of applied load and displacement, and the microscopic, heterogeneous and often non-continuum reality of material structure and behaviour. This bridge can take the form of a genuine interface between material and environment, e.g. at a surface, or can be a virtual one where the differing philosophies of design have to be merged. The interface has particular importance in circumstances where environmental influences have a key role in determining performance characteristics (e.g. creep, environmentally-assisted cracking, or corrosion), where performance is dominated by fatigue or fracture, where welding is used to join components, or where tribology plays a role. The paper focuses on the problems associated with cracking and uses case study examples drawn from engineering practice to illustrate the role of metallurgical factors in mechanical performance of materials.
\end{abstract}

KEYwORDS. Continuum mechanics; non-continuum materials; metallurgical parameters; case studies; fatigue; fracture; environment.

\section{INTRODUCTION}

$\mathrm{H}$ istorically, the education of mechanical engineers has had a strong focus on continuum mechanics as a tool for analysing the behaviour of structures and materials. In particular, solid mechanics (dealing with the behaviour of uncracked bodies subject to external load or deformation fields) and fracture mechanics (dealing with cracked bodies) have been very powerful in advancing our ability to calculate internal stresses and strains and to make realistic life assessments for engineering artefacts. The key assumptions in solid mechanics are usually that the body is a homogeneous, isotropic continuum, and considerable complexity is introduced into analysis when more complex situations arise.

Analytical complexity is also introduced as the applied loading or deformation field becomes more realistic; consider, for example, the increase in rigour necessitated by a move from unidirectional loading to triaxial constraint which occurs in the presence of a crack. Three dimensional stress states can be resolved into deviatoric (which promote shear deformation) and hydrostatic (which resist plastic deformation and promote fracture) stress components. As both plasticity and fracture are fundamentally controlled by inhomogeneous, non-isotropic and non-continuum materials 
behaviour it might be anticipated that prediction of structural behaviour would generally be difficult and imprecise. Several factors serve to mitigate these perturbations; one is the fact that plastic deformation is largely a constant volume process in which Poisson's ratio is 0.5 and this tends to promote $2 \mathrm{D}$ stress states, whilst a second is the local reduction in stress concentration factors that arises from plastic flow. However, increase in component or structural size tends to promote 3D stress states and increase plastic constraint (the move from plane stress to plane strain situations).

Another important factor is the relative size of critical defects at fracture compared with zones of local plasticity. When plasticity is very extensive the design criterion is likely to be plastic collapse of the structure and the analysis can be greatly simplified. Where plasticity is limited fracture will dominate and material behaviour will reflect interactions between small scale yielding, metallurgical factors (e.g. crystal structure; slip behaviour; grain and phase dimensions; inclusion type, distribution and size; heat treatment and hardness; surface condition; residual stress state). This is the realm of fracture mechanics, dislocation mechanics and plasticity theory. Many analytical and numerical approaches are approximate and the concepts of uncertainty, statistics and reliability have to be factored into the analysis.

The fundamental role of plasticity in cracking of metallic alloys has led to the adoption of a two parameter approach to predicting fracture using a Failure Analysis Diagram (FAD), which was developed for the CEGB R6 document, Assessment of the Integrity of Structures containing Defects [1], and has been adopted in similar British and European guidance for engineering critical assessment of defects [e.g. 2]. The FAD approach conjointly considers the susceptibility of a component or structure to fracture and to plastic collapse and plots this state on a failure locus diagram.

Thus, in considering the behaviour of materials under applied load or deformation, full consideration has to be given to the fact that their macroscopic behaviour reflects their microscopic composition and condition (heat treatment, hardness, defect population), the environment in which they operate (temperature, chemical species, contact with other parts), their surface condition (roughness, chemical composition, residual stress state) as well as the mode, frequency and complexity of the applied field (e.g. proportional or non-proportional multiaxial loading). The prediction and understanding of service cracking in materials is truly a cutting edge interface between metallurgy, materials science and mechanics.

This paper will focus on the metallurgical-mechanics interface in relation to cracking problems in metals at ambient temperature. Although non-metallic materials are both interesting and important, it remains true that the bulk of mechanical engineering practice is concerned with the behaviour of metals. It is also the case that failure by cracking at ambient temperatures is often experienced as a sudden and unforeseen phenomenon, whilst failure by corrosion, wear or creep mechanisms is time-dependent and the damage can often be monitored in service allowing repair and replacement to be properly scheduled. Sub-critical cracks may grow very slowly via fatigue or environmentally-assisted cracking, but once a critical crack size is reached (which may be either relatively small in terms of the scale of the structure, or in a position that is difficult to inspect) fast fracture occurs in metals at speeds which can reach around Mach 0.4 [3].

\section{CRACK TIP STRESS FIELDS IN THE PRESENCE OF PLASTICITY}

1 he first issue worth briefing considering concerns the perturbations imposed on the elastic stress field driving crack growth by the presence of a plastic enclave surrounding the crack. These perturbations are relatively minor in many circumstances but underpin the continuing controversy surrounding plasticity-induced shielding during fatigue crack growth. An increasing emphasis on multi-parameter characterisation of crack tip stresses, to give either more accurate K-solutions or improved fatigue life prediction models, brings into sharper focus the question of whether the elastic model for crack tip stresses can be improved to include influences arising from plasticity.

It was noted by Griffith [4] in a classic paper that the phenomenon of rupture in solids was governed by energy considerations, and that fracture occurred when the energy released by crack extension was equal to the energy absorbed during crack growth. This approach explicitly links crack size, applied stress and the concept of a material's resistance to fracture (with a magnitude equal to the value of the critical strain energy release rate $G_{C}$ ), but was difficult to extend to cracking of structures because of the complexity in quantifying and measuring the work to fracture. Nonetheless, it provides the beginning of a sound understanding of the driving force for cracking. Other important work in the early part of the $20^{\text {th }}$ century was the analysis of crack tip stress fields performed by Inglis [5] and Westergaard [6].

These stress and energy strands were brought together to lay the foundations of fracture mechanics in the work by Irwin in the 1950's on crack tip stress fields [7]. He recognised that the intensity of the applied stress field near the tip of crack was governed by the first term in a series expansion, through a $1 / V_{r}$ dependency where $r$ is the distance of the stressed element from the crack tip. This led to defining the numerator in this first stress term $\left(\sigma \sqrt{ }_{\mathrm{a}}\right)$ to be the 'stress intensity factor, $K$ ' with fracture occurring at a critical material constant value $K_{C}$ appropriate to the particular state of stress in the specimen. The stress intensity factor is used in situations where the overall stress-strain constitutive behaviour of the 
cracked body is linear and elastic, i.e. where there is a limited extent of crack tip plasticity (which arises from the high local stress concentration at the crack tip). Critical values of stress intensity factor and strain energy release rate are equivalent, as recognised in the case of plane stress by the equation $G=K^{2} / E$ where $E$ is the elastic modulus of the material. In plane strain this relationship becomes $G=K^{2} /\left(1-v^{2}\right) E$.

Linear elastic fracture mechanics and the stress intensity approach have proven very useful in characterising critical states in cracked and stressed structures with an associated decoupling of local crack tip microstructure and plasticity controlled events from the bulk applied elastic field. Plasticity is primarily considered through its influences on specimen compliance (via the Irwin plastic zone correction to apparent crack length), stress state and through the stipulation that nominal stress levels should be a relatively low fraction (perhaps $<0.6$ ) of the yield strength of the alloy. Paris [8] extended the use of the $\mathrm{K}$ concept to fatigue crack growth rate on the basis that if critical crack growth under monotonic loading was governed by the stress intensity factor, then sub-critical crack growth under cyclic loading may be governed by the range of the applied stress intensity, $\Delta K=K_{\max }-K_{\min }$. With the development of fracture mechanics into a mature discipline, the area of fatigue crack growth and life prediction under cyclic loading has become the prime application area for stress intensity approaches.

Extremely interesting (and largely ignored) questions remain as to why, and how, an elastic approach to crack growth is able to provide an adequate characterisation of phenomena that explicitly arise from plastic deformation processes. Satisfactory answers to these questions would seem likely to assist in resolving a number of controversies [9] associated with plasticity-induced shielding of the crack tip during fatigue crack growth [10,11]. The explanation advanced in recent work on this issue [12-14] considers the boundary conditions that would be imposed on the applied elastic field by a plastic 'inclusion' representing the plastic enclave surrounding the crack. These arise from crack wake contact (so-called plasticity-induced closure) and compatibility-induced stresses at the elastic-plastic interface. Plastic deformation is a constant volume process with Poisson's ratio $\nu=0.5$, whilst in elastic deformation $\nu \approx 0.3$ and plasticity-induced shear stresses must exist at the interface. The CJP model $[12,14]$ for crack tip stresses leads to a modified stress intensity driving crack growth forwards $K_{F}$, a retarding stress intensity $K_{R}$ which captures wake contact influences ahead of the crack tip, and a shear-induced stress intensity $K_{S}$. Analysis of its capability in characterising near-tip fringe patterns for difference in principal stresses [14], using full-field photoelastic data, indicate a lower fitting error than either two- $(K$ plus T-stress) or four-term solutions to the Westergaard or Williams [15] equations.

The stress variant of the equation representing the CJP model is given below along with 2-term and 4-term equations for the Williams solution. The Williams solution for 2-terms, $K$ and $T$-stress is:

$$
\frac{N f}{h}=\left|\sigma_{y}-\sigma_{x}+2 i \sigma_{x y}\right|=A z^{-\frac{1}{2}}+B z^{-\frac{3}{2}} \bar{z}+C z^{0}
$$

where the two unknown coefficients are $\mathrm{A}(\mathrm{A}+\mathrm{B}=0)$ and $\mathrm{C}$ and $\mathrm{z}$ is the coordinate of a stressed point in the complex plane. The 4-term solution is:

$$
\frac{N f}{h}=\left|\sigma_{y}-\sigma_{x}+2 i \sigma_{x y}\right|=\left|A\left(z^{-\frac{1}{2}}-z^{-\frac{3}{2}} \bar{z}\right)+B\left(z^{\frac{1}{2}}-z^{-\frac{1}{2}} \bar{z}\right)+C z^{0}+D(z+\bar{z})\right|
$$

where the four unknowns are A, B, C and D, while the CJP model is given as:

$$
\frac{N f}{h}=\left|\sigma_{y}-\sigma_{x}+2 i \sigma_{x y}\right|=\left|A z^{-\frac{1}{2}}+B z^{-\frac{3}{2}} \bar{z}+C z^{0}+D z^{-\frac{1}{2}} \ln z+E z^{-\frac{3}{2}} \bar{z} \ln z\right|
$$

where the four unknowns are $\mathrm{A}, \mathrm{B}, \mathrm{C}$ and $\mathrm{D}$ and $\mathrm{D}+\mathrm{E}=0$. This model allows for calculation of a stress intensity driving

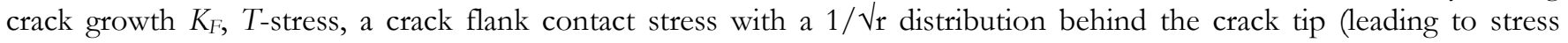
intensity acting to retard crack growth $K_{R}$ ) and a compatibility-induced shear stress as a boundary condition at the elasticplastic interface (leading to a $K_{S}$ term). This provides fringe patterns for difference in principal stresses which are directly comparable with photoelastic data.

Considering displacement fields, both perpendicular and parallel to the crack face, the Muskhelishvili complex stress function is given by:

$$
2 \mu(u+i v)=\chi \phi(z)-z \overline{\phi^{\prime}(z)}-\overline{\psi^{\prime}(z)}
$$

Displacement fields can be substituted into Eq. 4 to give: 


$$
\begin{aligned}
& 2 \mu(u+i v)=\chi\left(-2(B+2 E) z^{\frac{1}{2}}+4 E z^{\frac{1}{2}}-2 E z^{\frac{1}{2}} \ln z-\frac{C-F}{4} z\right) \\
& -z\left(-(B+2 E) \bar{z}^{-1 / 2}-E \bar{z}^{-1 / 2} \overline{\ln z}-\frac{C-F}{4}\right) \\
& -\left(A \bar{z}^{1 / 2}+D \bar{z}^{1 / 2} \overline{l n z}-2 D \bar{z}^{1 / 2}+\frac{C+F}{2} \bar{z}\right)
\end{aligned}
$$

where:

$$
\begin{array}{ll}
\mu & =\text { shear modulus, } \mathrm{MPa} \\
\chi & =\text { function of Poisson's ratio } \nu \\
u, v & =\text { horizontal and vertical displacements } \\
\phi(z), \psi(z) & =\text { auxiliary functions in Muskhelishvili‘s model } \\
\mathrm{z} & =\text { complex coordinate in the plane } \mathrm{z}=\mathrm{x}+\mathrm{iy} \\
A, B, C, D, E, F & =\text { unknown coefficients and } \mathrm{D}+\mathrm{E}=0
\end{array}
$$

Casting the CJP model in these terms allows direct comparison with full field crack tip displacement fields measured, for example, using digital image correlation techniques (Fig. 1). Expressions can be directly obtained from Eq. 5 giving $K_{F}$, $K_{S}, K_{R}$ and the $T$-stress as follows (note that when using displacement data rather than stress data, the $T$-stress has to be found as components $T_{x}$ in $\mathrm{x}$-direction and $T_{y}$ in $\mathrm{y}$-direction):

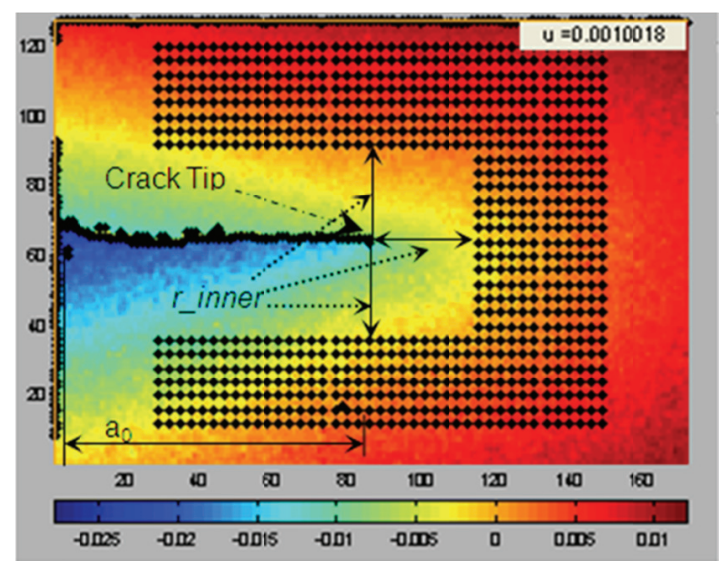

Figure 1: Displacement field around a crack tip measured using DIC techniques. The pattern of points is used for the full-field fitting between model and experimental data. A $2 \mathrm{~mm}$ thick aluminium specimen is used which contains a crack $29.3 \mathrm{~mm}$ long under a load of $120 \mathrm{~N}$. The measurement region is $17.7 \mathrm{~mm}$ by $13.2 \mathrm{~mm}$.

$$
\begin{aligned}
& K_{I}=\lim _{r \rightarrow 0}\left[\sqrt{2 \pi r}\left(\sigma_{y}+2 E \ln (r)\right)\right]=\sqrt{\frac{\pi}{2}}(A-3 B-8 E) \\
& K_{S}=\lim _{r \rightarrow 0}\left[\sqrt{2 \pi r}\left(\sigma_{x y}\right)\right]=\mp \sqrt{\frac{\pi}{2}}(A+B) \\
& K_{R}=\lim _{r \rightarrow 0}\left[\sqrt{2 \pi r}\left(\sigma_{y}\right)\right]=\sqrt{\frac{\pi^{3}}{2}}(D-3 E) \\
& T_{X}=-C \\
& T_{y}=-F
\end{aligned}
$$

It is not yet clear whether the CJP model, based on the plastic inclusion concept, will provide a means of characterising plasticity-induced growth rate perturbations, or lead to a reconciliation of conflicting views of plasticity-induced crack tip 
shielding. It does, however, capture influences of plasticity that have, up to now, largely been ignored and gives a lower fitting error when compared with photoelastic full-field fringe data.

\section{INTERGRANULAR FATIGUE}

I ntergranular faceting during fatigue is a second plasticity-induced phenomenon that occurs infrequently in specific alloys, e.g. certain $\alpha$-irons and ultra-low carbon steels with a body-centred cubic (bcc) crystal structure, and is a direct consequence of crystal structure and dislocation movement [16]. Slip mechanisms in bcc alloys are strongly dependent on temperature, strain rate and interstitial atom content and are governed by the behaviour of long screw dislocations, which are less mobile than non-screw dislocations and which make cross-slip more difficult. The core structure of a $1 / 2[111]$ screw dislocation in a bcc alloy is non-planar, lying in three $\left\{\begin{array}{llll}1 & 1 & 1\end{array}\right\}$ planes (Fig. 2). It spreads into three $1 / 6\left[\begin{array}{lll}1 & 1 & 1\end{array}\right]$ fractional dislocations which also spread asymmetrically on three $\left\{\begin{array}{lll}1 & 1 & 2\end{array}\right\}$ planes in the twinning sense [18]. This leads to slip asymmetry whereby the shear stress to move a dislocation in one direction on a slip plane is not the same as that required to move it in the opposite direction on the same plane.

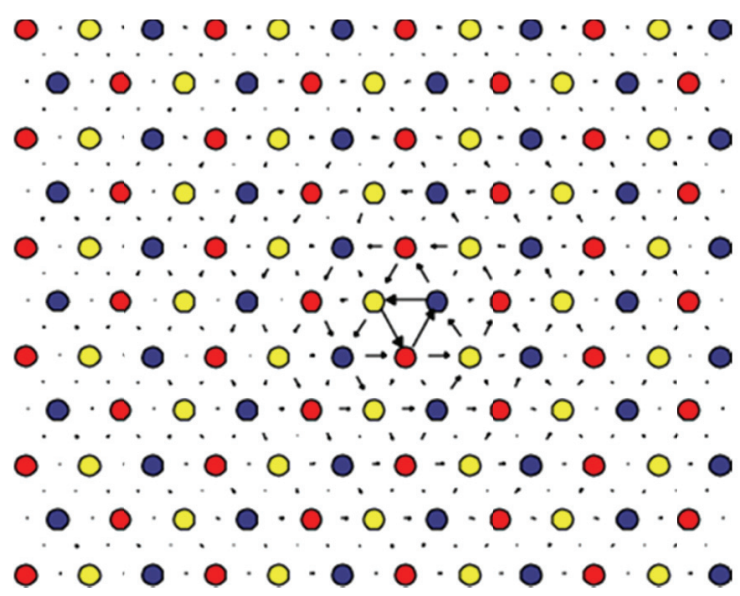

Figure 2: Core of a $1 / 2[111]$ screw dislocation parallel to the plane of the figure. The atomic block is formed by periodically repeating the three (111) layers (distinguished by different coloured atoms) along the dislocation line.

The arrows are the relative (screw) displacements of neighbouring atoms along the dislocation line [17].

The motion of screw dislocations hence depends on stress-assisted thermally activated generation of kink pairs on $\left\{\begin{array}{lll}1 & 1 & 0\end{array}\right\}$ and $\left\{\begin{array}{lll}1 & 1 & 2\end{array}\right\}$ slip planes. Interstitial atoms present in the cores of screw dislocations apparently make this process easier [19] and ultra-low interstitial atom content (e.g. C, B, N) would therefore promote slip asymmetry in bcc alloys. This slip asymmetry gives rise to shape changes of bcc grains undergoing fully reversed cyclic deformation. Stress concentrations can then form along grain boundaries which favours the nucleation of intergranular fatigue cracks. During subsequent growth along extended crack paths plastic deformation may be strongly localised within grain boundaries, particularly in susceptible alloys subjected to fully reversed loading in high cycle fatigue where levels of plastic strain range are low.

An interesting example of this phenomenon occurs in interstitial-free (IF) steels which constitute one of the major groups of bcc steels used in the automotive industry for forming thin gauge sheet body panels. In performance terms, these alloys have to balance several conflicting requirements, i.e. deep-drawing capability, fatigue resistance, tensile strength and light weight and are likely to be subject to reversed cyclic loading in service. The conflict in requirements arises because increased formability and deep-drawing capability are assisted by very low amounts of interstitial elements such as carbon, boron and nitrogen, typically $10-200 \mathrm{wt} \mathrm{ppm}(0.001 \mathrm{wt} \%$ to $0.02 \mathrm{wt} \%)$. At the small plastic strains typical of high cycle fatigue loading, however, low interstitial content $(<40 \mathrm{ppm})$ promotes IG cracking through the slip asymmetry mechanism outlined above. IG cracking may also be promoted with high strength IF steel grades containing increased levels of $\mathrm{P}$ and $\mathrm{Mn}$ in solid solution. Susceptibility to brittle fracture has been shown to be higher in the presence of increased $\mathrm{P}$ and lower interstitial C, B or N [16].

In the case of IF steels, despite plastic deformation being strongly localised at grain boundaries, which leads to IG faceting particularly during crack initiation and long life fatigue, the fatigue performance is little different to other comparable 
alloys which do not exhibit such IG faceting during crack initiation and growth [16]. This is unusual, as the presence of IG facets on a fracture surface are usually an indication that some form of embrittlement has occurred and that resistance to either critical or sub-critical cracking would be lowered. Fig. 3 shows typical IG faceting observed in these alloys.

\section{RESIDUAL STRESSES AND WELDS}

$\mathrm{R}$ esidual stresses arise as a consequence of misfit between various regions [20] and are hence an inescapable consequence of manufacturing and fabrication processes, with magnitudes that locally are often a high proportion of the yield or proof strength. These regions of misfit can occur with several different size scales and hence different distances over which the residual stresses self-equilibrate. Their influence on mechanical performance of alloys therefore also varies with type, nature and origin [20]. Type I macrostresses can affect the performance of structures in a number of ways, including changes to load eccentricity and failure stress during buckling, and influences on the fracture stress and fatigue crack growth. These stresses often arise from non-uniform plastic deformation or from assembly misfit of structural parts. They also occur as a consequence of the crack growth process itself (crack tip plastic zone and crack wake effects, local phase transformations), and are particularly influenced by thermal processes (e.g. heat treatment or welding), mechanical properties, microstructure and geometry of the component or part.

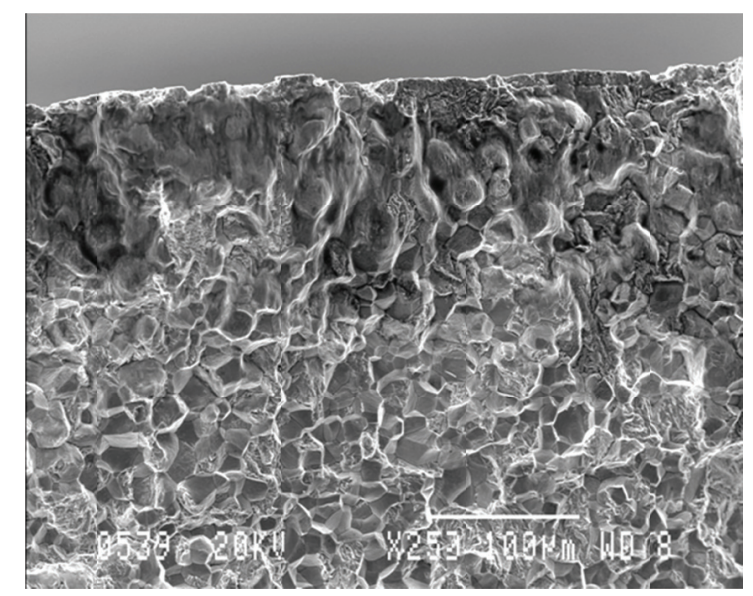

Figure 3: IG faceting near the crack initiation site in an IF steel containing $0.001 \mathrm{C}, 0.380 \mathrm{Mn}, 0.004 \mathrm{Si}, 0.008 \mathrm{P}, 0.005 \mathrm{~S}, 0.018 \mathrm{Nb}$ and $0.013 \mathrm{Ti}$. The steel has a yield strength of $174 \mathrm{MPa}$.

Their magnitudes can be highly variable; both from place-to-place within the component, and between components or structures produced under nominally similar conditions. The external application of cycles of load, displacement or heat, particularly in the presence of stress concentrators such as weld toes, defects and cracks, leads to significant changes in the residual stress profiles during service [21]. Compressive residual stresses are routinely used to enhance service performance through, for example, peening [22] or cold-working techniques, which are often considered as improving the fatigue 'quality' of the component [23]. Type II stresses are intergranular in scale and influence aspects of performance such as the initiation of fatigue cracks and corrosion processes. Type III stresses are of the order of atomic size and arise from interface coherency, e.g. of intermetallic particles and regions, and from dislocation stress fields, and influence plastic slip processes. Despite the widespread existence and frequent use of residual stresses in enhancing performance under cyclic loading, there is still a lack of accurate $3 \mathrm{D}$ information on the magnitude and re-distribution (or relaxation) under load.

Welding is the most commonly used structural joining process and the residual stress distribution assumed in defect assessment for welded joints often has a deciding influence on the analysis outcome [24]. Thus fatigue design codes tend to propose very conservative ways of dealing with residual stress in fracture mechanics assessments of remanent life or engineering criticality of defects. A large contribution to this uncertainty arises from data limitations associated with surface measurements, e.g. using low energy X-ray sources, imprecision in hole drilling parameters and relatively small sets of measurements, alongside an 'innate' contribution to scatter from the welding process [24].

From the 1970s onwards, the available X-ray intensity increased dramatically as synchrotron light sources were developed. A synchrotron light source is a form of particle accelerator in which particles (usually electrons) are initially accelerated in 
a linear accelerator and then injected into a storage ring where they are made to circulate via magnetic fields and accelerated (to about 0.6x the speed of light, equivalent to an energy of $6 \mathrm{GeV}$ ) in an electric field. Both fields are carefully synchronized with the travelling particle beam. Third-generation synchrotron X-ray sources are designed around multi-magnet insertion devices ("undulators" and "wigglers") in straight sections of the storage ring which bend the electron beam and convert some of its energy into high energy electromagnetic radiation ('hard' or high energy X-rays see Fig. 4 - with energies in the range of $20-400 \mathrm{keV}$ ).

Synchrotron radiation is very suitable for fast, accurate residual stress measurements through up to about $10 \mathrm{~mm}$ of steel. Its advantages derive from:

$\checkmark$ High brightness and high intensity, many orders of magnitude greater than with conventional X-rays X-ray tubes

$\checkmark$ High collimation, i.e. small angular divergence of the beam

$\checkmark$ Low emittance, i.e. the product of source cross section and solid angle of emission is small

$\checkmark \quad$ Wide tunability in energy/wavelength

$\checkmark$ High brilliance (photon flux)

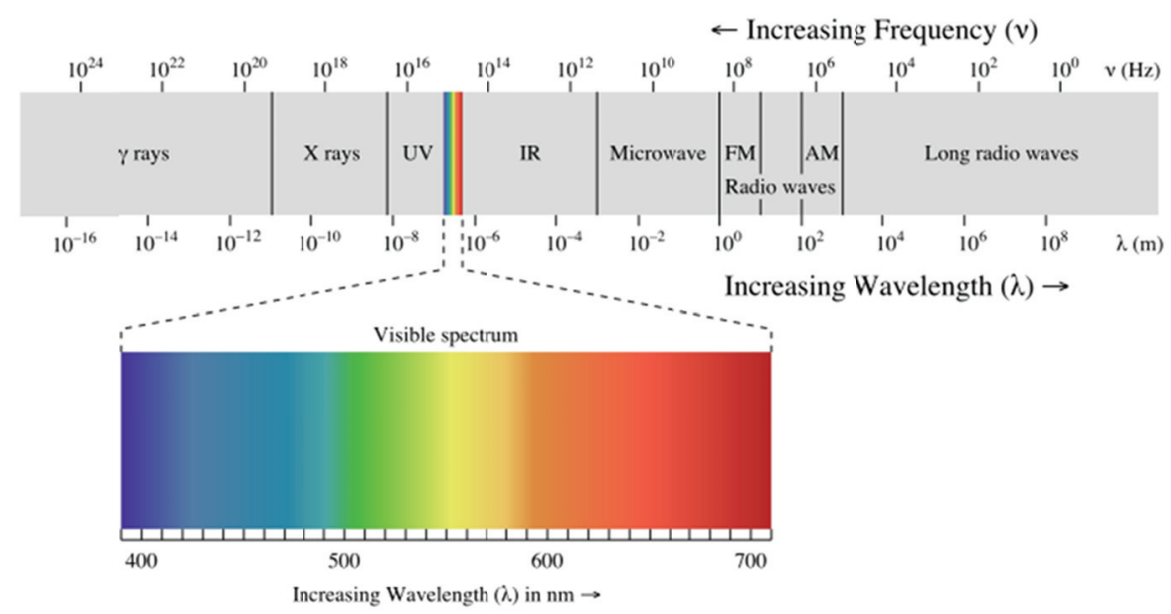

Figure 4: Electromagnetic spectrum showing the region of visible light [25].

These attributes, coupled with greatly improved data analysis software and automated 3D stages (with precision of spatial location $\sim 10 \mu \mathrm{m}$ ) capable of accommodating large and heavy components, allow large sets of strain data to be acquired in a typical experiment extending over several days.

Neutron diffraction is the other widely used technique capable of yielding data through thick specimens (up to $\approx 50 \mathrm{~mm}$ of steel). The required free neutrons can be obtained in a nuclear reactor where they are set free through nuclei decay, particularly when fission occurs. All quantum particles can exhibit wave phenomena such as diffraction, which occurs when waves encounter an array of obstacles whose size is comparable with the wavelength. A beam of neutrons emanating from a reactor can be slowed down and selected by their speed such that their wavelength lies in a range from $0.1 \AA$ to $1000 \AA$, making them an ideal probe of atomic and molecular structures, either in the form of single atomic species or complex biopolymers. One Angström $(0.1 \mathrm{~nm})$ is the typical separation between atoms in a solid crystalline material. Such a neutron beam can then be used to perform a diffraction experiment; in impinging on a crystalline sample it will scatter under a limited number of well-defined angles according to the same Bragg's law that describes X-ray diffraction. Neutron and synchrotron diffraction techniques can provide complementary information:

\section{Neutrons}

Particle beam (neutral subatomic particle)

Interactions with the nuclei and the magnetic moment of unpaired electrons (in the sample)

Scattered by all elements, also the light ones like the hydrogen isotopes

Deep penetration depth (bulk studies of samples)

Less intense beam measuring larger samples

\section{Synchrotron radiation}

Light beam (electromagnetic wave)

Interactions with the electrons surrounding the nuclei (in the sample)

Mainly scattered by heavy elements

Small penetration depth (surface studies of samples)

Very intense beam measuring small or ultra-dilute samples 
Applications:

$\checkmark$ Magnetic structures and excitations

$\checkmark$ Organic structures using the H-D isotope effect

$\checkmark$ Bulk studies (strains, excitations)

$\checkmark$ Low-energy spectroscopy, e.g. molecular vibrations
Applications:

$\checkmark$ Protein-crystal structures

$\checkmark$ Fast chemical reactions

$\checkmark$ Surface studies (defects, corrosion)

$\checkmark$ High-energy spectroscopy, e.g. measurements of electron energy-levels

Similar improvements to those found with synchrotron radiation sources, in automated sample positioning and data analysis for high intensity neutron sources, mean that residual strain and stress data can be obtained over a wide range of scales for most metallic alloys. It is now also possible to apply in-situ loading to specimens whilst they are mounted on the neutron or synchrotron instrument.

The utility of neutron diffraction is easily demonstrated; Fig. 5 shows residual stresses being measured in stainless steel welds on the sophisticated 6-axis hexapod Stewart platform of the SALSA neutron diffraction instrument at the Institut Laue-Langevin in Grenoble, France. This experiment was concerned with assessing the influences of overlay repair welding using autogenous friction stir welding and laser beam welding with 316L and nickel-based powder consumables. These repair welds were made in annealed SA240 Grade 304L austenitic stainless steel plate $10 \mathrm{~mm}$ thick and were superimposed on conventional V-preparation butt welds. They would be expected to display better corrosion resistance than the parent plate. The original butt welds were made using either submerged arc or manual metal arc welding processes, simulating the original construction methods used in the stainless steel tanks. Stainless steel can experience stress corrosion cracking (SCC) during service in the power generation industry, which is driven by high levels of residual stress, and it is clearly important to know how repair welding procedures will affect the residual stress distribution. Details of these various welds are shown in Fig. 6. It is worth noting that texturing in austenitic weld metals can lead to weak diffraction intensities, requiring long count times for each measurement [26].

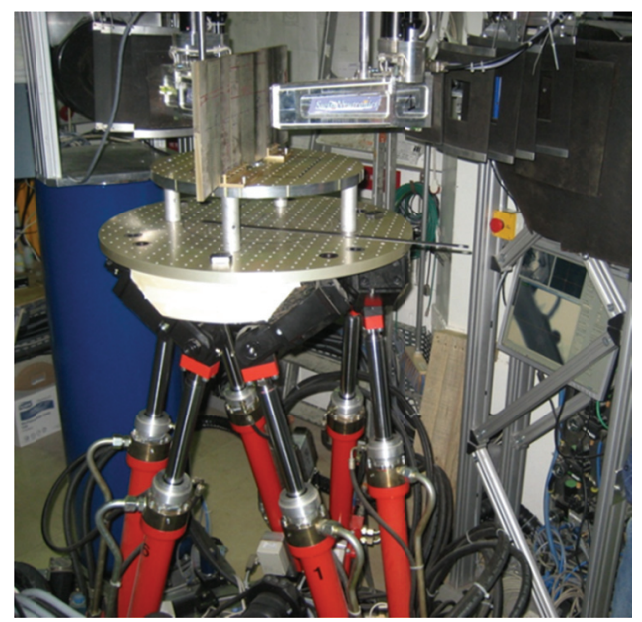

Figure 5: Measurement of residual stresses in $16 \mathrm{~mm}$ thick plates of 304L stainless steel.

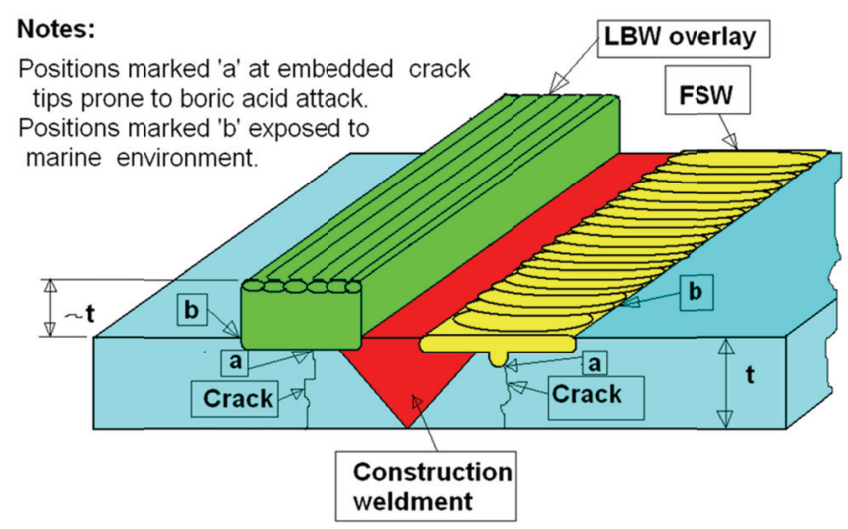

Figure 6: Illustration of repair weld procedures superimposed on SCC cracks in the heat-affected zone (HAZ) of fusion welds in $304 \mathrm{~L}$ stainless steel. 
Neutron diffraction data were measured using the $\left\{\begin{array}{lll}3 & 1 & 1\end{array}\right\}$ planes in the alloy giving a diffraction angle of $91.35^{\circ}$, vertical and horizontal slit sizes of $1.1 \mathrm{~mm}$, and a neutron wavelength of $1.5488 \AA$. The typical sample set-up for measuring strains in the direction transverse to the weld is shown in Fig. 7.

The 3D stress state was measured with a spacing of $2 \mathrm{~mm}$ from the centre-line of the weld at a depth of $1 \mathrm{~mm}$ and with a spacing of $4 \mathrm{~mm}$ at depths of $2 \mathrm{~mm}, 5 \mathrm{~mm}$ and $8 \mathrm{~mm}$ into the plate. Residual stress data for specimen 3 (laser beam overlay weld superimposed on a friction stir weld) are shown as contour maps in Fig. 8a, whilst Fig. 8b gives the residual stress profiles in the three coordinate directions measured at a depth of $1 \mathrm{~mm}$ below the surface of the overlay. The highest tensile residual stresses in the overlay sample occur at about $5 \mathrm{~mm}$ to the side of the centre-line of the original Vbutt weld, where the FS weld was located. Peak stresses in the repair overlay are lower than those occurring in the original site welds made in the stainless steel plate (Fig. 9), whilst the corrosion resistance of the overlay alloy is higher than the 304L. The possibility of SCC should therefore be greatly reduced after this repair procedure has been applied. Heat treatment of the weld region would further reduce residual stresses and these can be re-measured after heat treating the specimen.

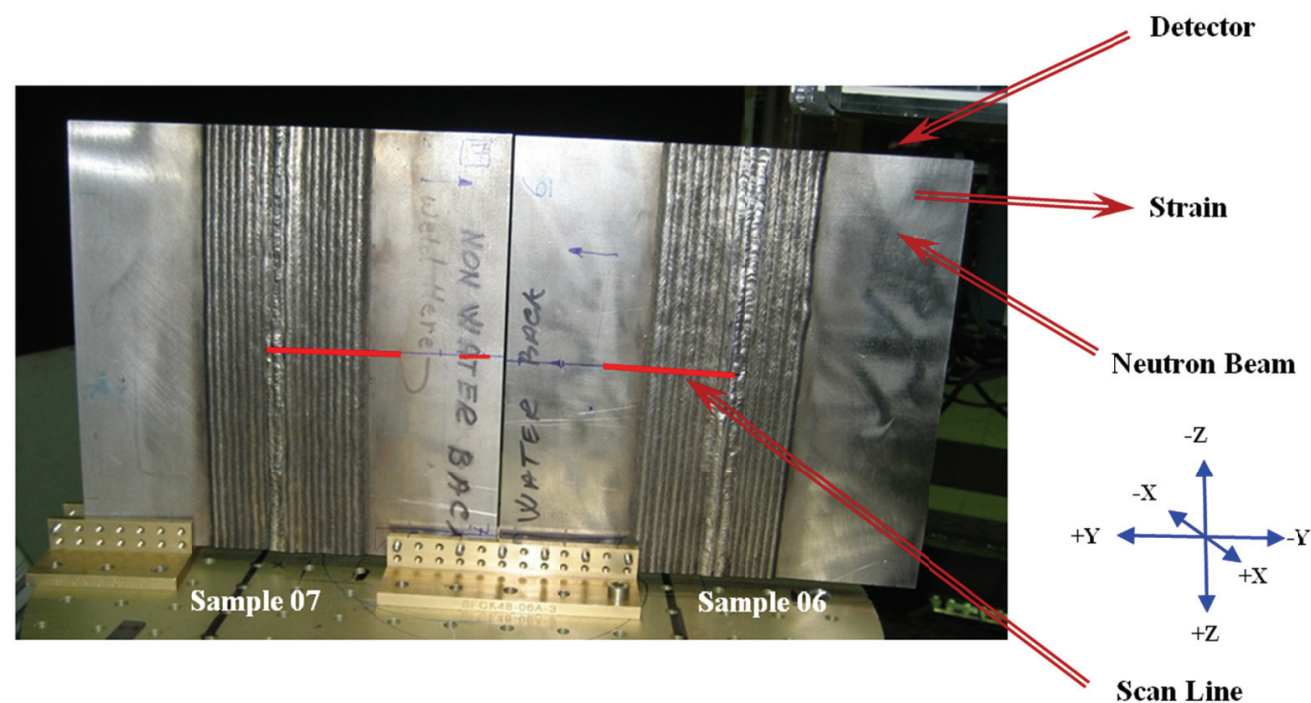

Figure 7: Two 304L stainless steel welds shown in position on the SALSA instrument for determination of the strains transverse to the weld.

SAMPLE 3

FSW and Laser Overlay on a Site Weld
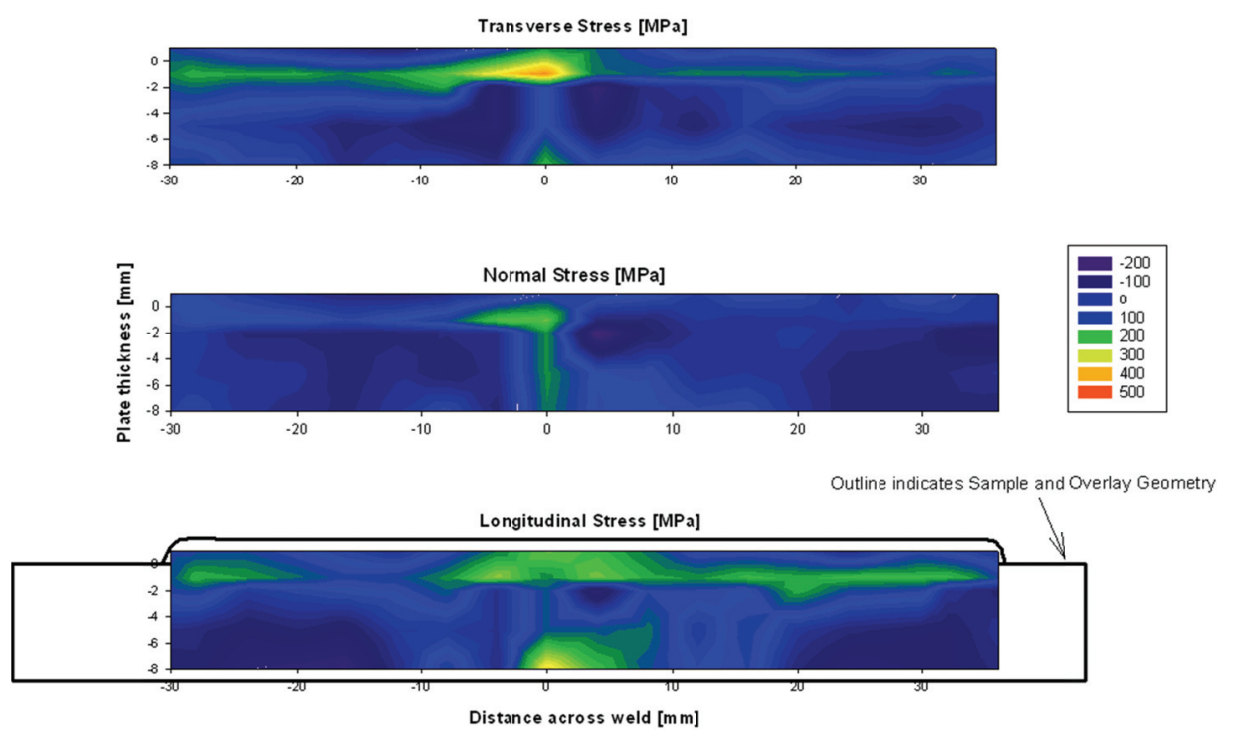

Figure 8a: Residual stress contour maps for FSW and laser beam weld overlay on a V-butt weld in 10 mm plate of 304L stainless steel. 
Sample 3 (Laser and FSW) - 1mm Depth

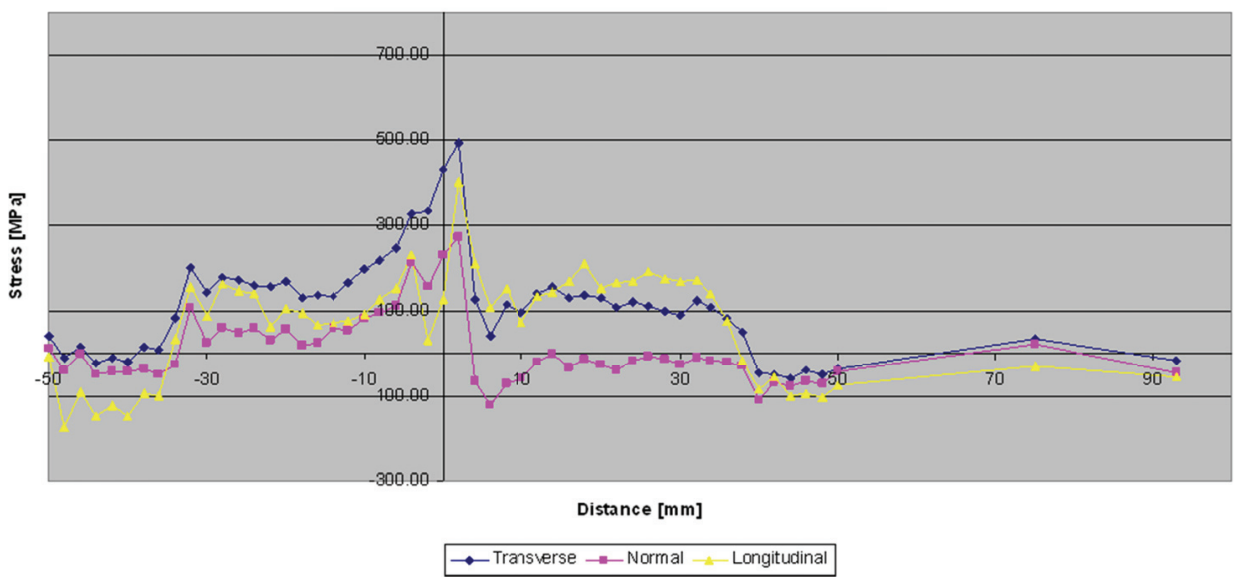

Figure 8b: Residual stress profiles at a depth of $1 \mathrm{~mm}$ below the surface of the weld overlay.

It is clear that neutron and synchrotron diffraction are very powerful techniques for assessing 3D residual stress fields in service welds and other components, particularly if the results are linked to finite element modelling and used as a calibration of the accuracy of the predictive model.

In measuring residual stresses using diffraction techniques, crystal lattice spacing is determined relative to the strain-free lattice spacing $d_{0}$, which is affected by factors such as composition and microstructure and hence can vary across a weld region. An important aspect of diffraction-based measurements is therefore determination of $d_{0}$, often by making measurements on toothcomb specimens cut across the weld region [27] or via use of small cubes (perhaps $2 \times 2 \times 2 \mathrm{~mm}$ ) cut from a number of relevant positions in the plate.

This brief discussion has, hopefully, indicated that not only are residual stresses an interesting demonstration of the interface between metallurgy and mechanics in materials performance, but also that their measurement and interpretation equally require an understanding of the effect of crystal structure and microstructure on continuum and physics phenomena.
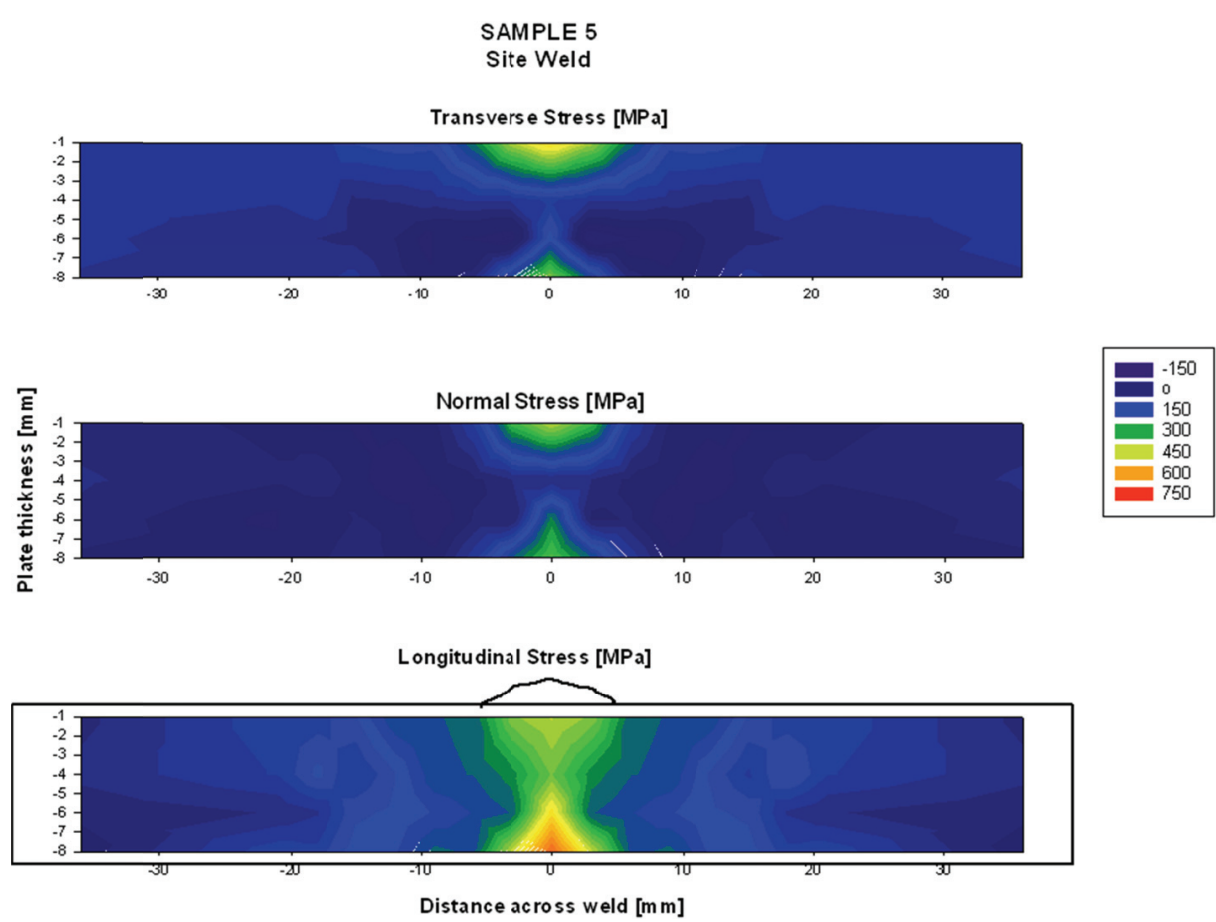

Figure 9: Residual stress contours for an original site weld made in the $10 \mathrm{~mm}$ 304L stainless steel plate. 


\section{CONCLUSIONS}

$\mathrm{T}$ his paper has presented a very brief overview of certain areas where the mechanical performance of metals is dominated or compromised by compositional, crystal structure or metallurgical factors, focussing particularly on plasticity and its influences. Its key premise has been that assuring structural integrity requires a sound understanding of the interface between, and synergetic effects of, mechanical engineering and materials science. In the experience of the author, over some 25 years of industrial consulting practice, most service failures arise from a poor understanding of this interface and of its design consequences. Some problems that occur at this interface are very subtle whilst others are glaringly obvious, but all of them can lead to unintended consequences if the reality of microscopic or mesoscopic material performance is ignored in a macroscopic continuum mechanics analysis of structures.

\section{ACKNOWLEDGEMENTS}

$\mathrm{T}$ he award of beam time from the ILL under experiment 1-01-73 is gratefully acknowledged, together with assistance from the beamline scientist, Dr D J Hughes. The indispensable assistance of Philip Doubell, Professor Danie Hattingh and Dr Axel Steuwer with this experiment is also duly acknowledged.

\section{REFERENCES}

[1] CEGB R6, Revision 4, Assessment of the integrity of structures containing defects, British Energy Generation Ltd, Gloucester, England, (August 2007).

[2] BS 7910:2005, Guide to methods for assessing the acceptability of flaws in metallic structures, British Standards Institution (2005). ISBN: 9780580601088

[3] J. G. A. de Graaf, Applied Optics, 3(11) (1963) 1223.

[4] A. Griffith, The phenomena of rupture and level in solids, Phil. Trans. Series, A 221 (1920)163.

[5] E. Inglis, Trans. Institution of Naval Architects, 55 (1913) 219.

[6] H. M. Westergaard, Proc. Am. Concrete Inst., 30 (1934) 93.

[7] G. R. Irwin, J. App. Mech., 24 (1957)361.

[8] P. C. Paris, R. E. Gomez, W. E. Anderson, The Trend in Engng, University of Washington, 13(1) (1961) 9.

[9] M. N. James, In: Procs 9th Intl Conf. on Fract., Sydney, (eds. B L Karihaloo et al), Australia, Pergamon Press, 5 (1997) 2403.

[10] R. H. Christensen, Appl. Mater. Res., 2 (4)(1963) 207.

[11] W. Elber, Engng Fract. Mech., 2 (1970) 37.

[12] C. J. Christopher, M. N. James, E. A. Patterson, K. F. Tee, Int. J. Fract., 148 (2007) 361.

[13] M. N. James, C. J. Christopher, Lu Yanwei, E. A. Patterson, In: Procs $2^{\text {nd }}$ Int. Conf. on Advances in Product Development and Reliability (PDR'2010), Shenyang, China, (2010); Advanced Mats Res., 118-120 (2010) 1.

[14] M. N. James, C. J. Christopher, Lu Yanwei, E. A. Patterson, In: Procs 6th Int. Conf. on Mats Struct. and Micromechanics of Fracture, Brno, Czech Republic, (2010). To be published in Key Engng Mats.

[15] M. L. Williams, J. Appl. Mech., 24 (1957) 109.

[16] M. N. James, Engng Fract. Mech. Mechanics, 77 (2010) 1998.

[17] http://cnls.lanl.gov/ groger/codes/ddplot/ddplot.html, accessed on 5 September 2009.

[18] D. Hull, D. J. Bacon, , Introduction to Dislocations, $3^{\text {rd }}$ Edition, Pergamon Press, Oxford (1984)

[19] A. Seeger, Phil. Mag. Letters, 84(2) (2004) 79.

[20] P. J. Withers, H. K. D. H. Bhadeshia, Mater. Sci. Technol., 17 (2000) 355.

[21] M. N. James, D. J. Hughes, Z. Chen, H. Lombard, D. G. Hattingh, D. Asquith, J. R. Yates, P. J. Webster, Engng Failure Anal., 14 (2007) 384.

[22] M. N. James, M. Newby, D. G. Hattingh, A. Steuwer, Procedia Engng, 2 (1) (2010) 441.

[23] M. Ofsthun, Int. J. Fatigue, 25 (2003) 1223.

[24] P. J. Bouchard, Int. J. Pressure Vessels and Piping, 85 (2008) 152.

[25] Wikipedia, Electromagnetic radiation, http://en.wikipedia.org/wiki/Electromagnetic_radiation, accessed on 24 June 2010. 
[26] S. Okido, K. Saito, H. Suzuki, Mats Sci. Forum, 524-25 (2006) 697.

[27] D. J. Hughes, M. N. James, D. G. Hattingh, P. J. Webster, J. Neutron Res., 11 (2003) 289. 